\section{The Effect of Early Postoperative Urinary Catheter Removal on Patient Satisfaction in Gynecologic Oncology}

\author{
Menzies A, Dayton S, Burke WM, Gossner G, Pearl ML,
}

Stony Brook Medicine

\section{Introduction}

Since 1990, healthcare industries have initiated movements toward quality improvement. Patient satisfaction has become an important aspect of healthcare quality management. ${ }^{6}$ It is difficult to compare satisfaction among hospitals because often each institution implements its own survey. There is no standardized survey instrument to assess patient satisfaction.

Patient satisfaction can be affected by age, gender, level of education, previous experiences in healthcare, and health status. Current data looking at the correlation between those categories and satisfaction has revealed mixed results. ${ }^{4}$ Patient satisfaction can be characterized by how the patient reacts to aspects of her hospital experience. ${ }^{1}$ This study will examine the postoperative management of urinary catheters in gynecologic oncology patients.

The Infectious Diseases Society of America agrees that there is strong evidence that indwelling catheters should be removed as soon as they are no longer required in order to reduce the risk of bacteriuria and UTI. Few studies,

however, have reviewed the patient's perspective on the indwelling catheter and how it affects overall satisfaction. ${ }^{2,4,7}$ There are no clear guidelines regarding the optimal time to remove the catheter. The decision is typically based on the surgeon's custom rather than evidence-based knowledge. ${ }^{3}$

In this quality improvement project, we will focus on the impact of the timing of urinary catheter removal on patient satisfaction in patients who have undergone major surgery on a gynecologic oncology service.

\section{Methods}

We conducted a prospective cohort QA study of 111 patients undergoing major surgery on a gynecologic oncology service between 2016-2018.

In the first cohort, urinary catheters were removed at $6 \mathrm{AM}$ on postoperative (POD) day \# 1. In the second cohort, the catheters were removed 4 hours after surgery. In both cohorts, a satisfaction survey was collected in the afternoon on POD \# 1. The primary outcome was patient satisfaction. We assessed for several secondary clinical outcomes including acute urinary retention, urinary tract infection, duration of hospital stay, timing of initiation of ambulation, and hospital readmission.

Participation in the survey was anonymous and voluntary.

\section{Exclusion criteria}

1. Procedure complications that required indwelling catheter to be kept postoperatively

2. Surgeon preference

3. Patient undergoing radical hysterectomy

4. Known history of neurological disorders affecting bladder control

Data analysis was performed utilizing univariate/bivariate analyses as well as T-test, Chi-square/Fisher's exact test, and one-way ANOVA.

\section{Patient Satisfaction Survey}

1. How do you feel at this time?

2. How did you sleep last night?

3. How would you rate your pain?

4. Do you feel nauseated?

5 . Would you like to walk in the hall this morning?

6. How did it feel having a urinary catheter in place?

7. How did having your urinary catheter removed feel?

8 . When was the urinary catheter removed?

9. How many times did you need to use the bedpan/commode/bathroom?

10. Did the hospital staff respond in a timely fashion to your request to use the bedpan/commode/bathroom?

11. How long did you have to wait to use the bedpan/commode/bathroom?

12. Describe your experience of using the bedpan/commode/bathroom?

13. Did you experience any pain or discomfort when urinating?

14. Would you have wanted your urinary catheter removed sooner?

15. Overall how would you describe your medical experience during this admission?

\section{Overall, how would you describe your medical experience during this admission?}

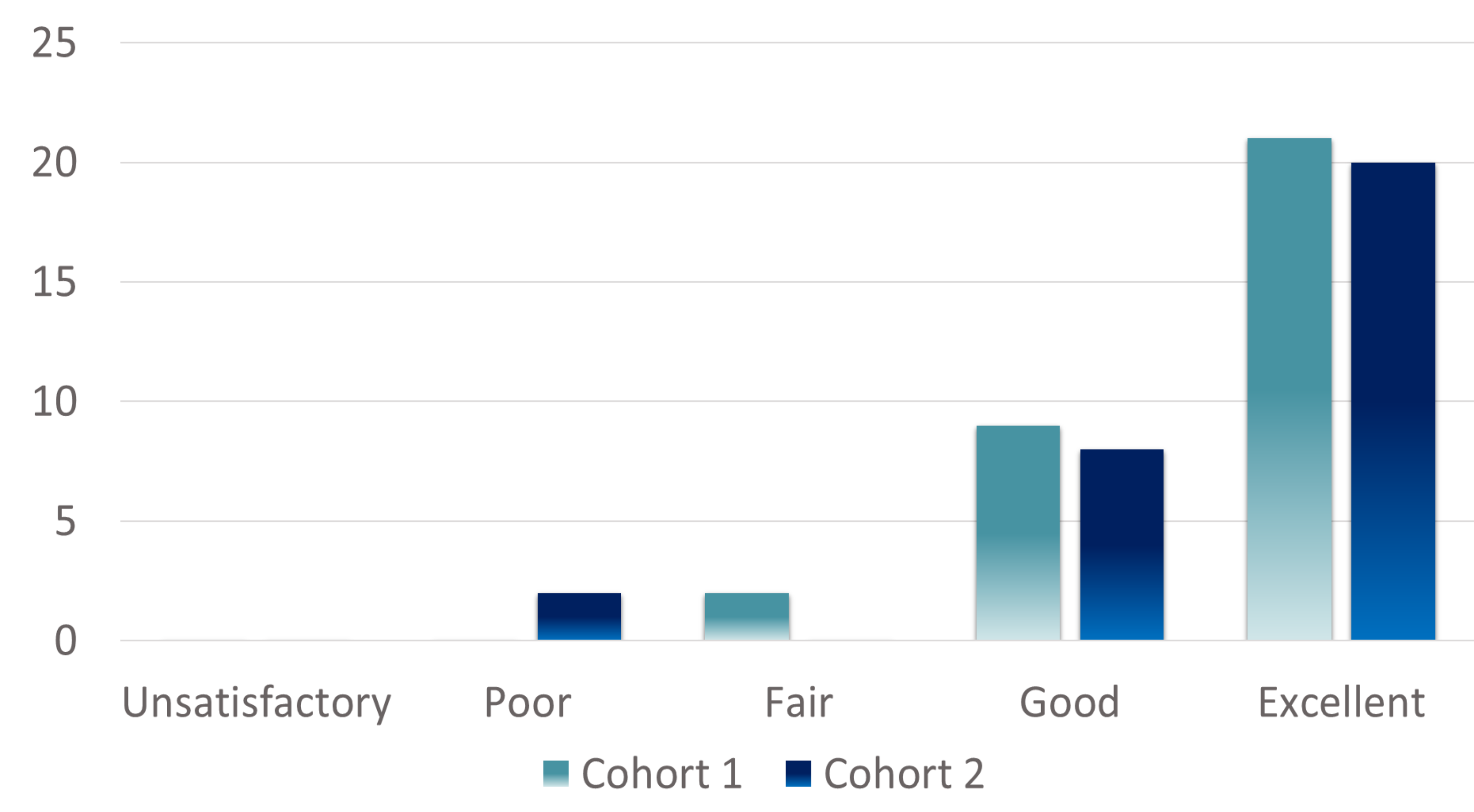

\begin{tabular}{|c|c|c|c|}
\hline & Cohort 1 & Cohort 2 & p \\
\hline Mean length of procedure $(\mathrm{min})$ & 155 & 134 & 0.1618 \\
\hline Mean estimated blood loss $(\mathrm{ml})$ & 244 & 220 & 0.5451 \\
\hline Mean time to void $(\mathrm{hr})$ & 3.4 & 4.3 & 0.0326 \\
\hline Mean length of hospital Stay $(\mathrm{hr})$ & 36 & 31 & 0.5411 \\
\hline
\end{tabular}

Secondary Clinical Outcomes

\begin{tabular}{|c|c|c|c|}
\hline \multicolumn{4}{|c|}{ Secondary Clinical Outcomes } \\
\hline & Cohort 1 & Cohort 2 & p \\
\hline Acute urinary retention & 1 & 0 & 0.4839 \\
\hline $\begin{array}{c}\text { Urinary complaints at postop visit } \\
\text { Positive urine culture within 30 days } \\
\text { after surgery }\end{array}$ & 5 & 2 & 0.692 \\
\hline $\begin{array}{c}\text { Hospital re-admission within 30 days } \\
\text { after surgery }\end{array}$ & 2 & 1 & 0.6299 \\
\hline
\end{tabular}

- Thirty-three patients (29.7\%) were excluded due to postoperative concerns, including hemodynamic instability and necessity for prolonged urinary catheterization.

The majority of patients described their hospital experience as good or excellent. There was no significan change in level of satisfaction between the two cohorts - Among the included 78 patients, only one experienced acute urinary retention. This patient was in the first cohor of the study.

- There was no significant difference in duration of hospital stay (1.29 vs. 1.38 days), urinary tract infections, and hospital readmissions

- In the first cohort, $43 / 44$ patients were ambulating by 1200 on POD\#1, while in the second arm 31/34 were ambulating by 0600 on POD\#1

\section{Conclusions}

- Patient satisfaction with early postoperative urinary catheter removal following major surgery on a gynecologic oncology service was not inferior to routine removal the following morning.

- Clinical outcomes including acute urinary retention, UTI, hospital readmission rates were not significantly different between the two cohorts

- Nearly all patients in the second cohort were ambulating on POD\#0.

- We recommend early urinary catheter removal unless otherwise indicated by postoperative concerns.

\section{References}

Ahmed, Magdy R., Waleed A. Sayed Ahmed, Khaled A. Atwa, and Lobna Metwally. "Timing of Urinary Catheter Removal after Uncomplicated Total Abdominal Hysterectomy: A Prospective Randomized Trial." European Journal of Obstetrics \& Gynecology and Reproductive Biology 176 (2014): 60-63. Web.

2. Al-Abri, Rashid, and Amina Al-Balushi. "Patient Satisfaction Survey as a Tool Towards Quality Improvement." Oman Medical Journal Oman Med J 29.1 (2014): 3-7. Web.

3. Alessandri, Franco, Emanuela Mistrangelo, Davide Lijoi, Simone Ferrero, and Nicola Ragni. "A Prospective, Randomized Trial Comparing Immediate versus Delayed Catheter Removal following Hysterectomy." Acta Obstetricia Et Gynecologica Scandinavica Acta Obstet Gynecol Scand 85.6 (2006): 716-20. Web.

4. Dunn, Terry S., Judith Shlay, and Dave Forshner. "Are In-dwelling Catheters Necessary for 24 Hours after Hysterectomy?" American Journal of Obstetrics and Gynecology 189.2 (2003): 435-37. Web.

5. Schoenfelder, T., J. Klewer, and J. Kugler. "Determinants of Patien Satisfaction: A Study among 39 Hospitals in an In-patient Setting in Germany." International Journal for Quality in Health Care 23.5 (2011) 503-09. Web.

6. Urden, Linda D. "Patient Satisfaction Measurement." Lippincott's Case Management 7.5 (2002): 194-200. Web.

7. Zhang, Peng, Wan-Li Hu, Bei Cheng, Long Cheng, Xiao-Kan Xiong, and Yang-Jun Zeng. "A Systematic Review and Meta-analysis Comparing Immediate and Delayed Catheter Removal following Uncomplicated Hysterectomy."International Urogynecology Journal Int Urogynecol J 26.5 (2014): 665-74. Web. 